$$
\begin{aligned}
& \text { Abbreviations: } \\
& \mathrm{PL}=\text { Plenary; } \mathrm{LO}=\text { Lightning oral } \mathrm{MP}=\text { Moderated poster; } \\
& \mathrm{P}=\text { Poster }
\end{aligned}
$$

*Corresponding authors are underlined.

\section{Plenary Oral Presentations}

\section{PL01}

Multicentre before-after implementation study of the Ottawa subarachnoid hemorrhage strategy

J. J. Perry, MD, MSc, M. L.A. Sivilotti, MD, MSc, M. Emond, MD, MSc, C. M. Hohl, MD, MHSc, H. Lesiuk, MD, J. Sutherland, MEd, M. Khan, MSc, K. Abdulaziz, MSc, G. A. Wells, PhD, I. G. Stiell, MD, MSc, University of Ottawa, Department of Emergency Medicine, Ottawa, ON

Introduction: The Ottawa SAH Rule was developed to identify patients at high-risk for subarachnoid hemorrhage $(\mathrm{SAH})$ who require investigations and the 6-Hour CT Rule found that computed tomography (CT) was $100 \%$ sensitive for SAH 6 hours of headache onset. Together, they form the Ottawa SAH Strategy. Our objectives were to assess: 1) Safety of the Ottawa SAH Strategy and its 2) Impact on: a) CTs, b) LPs, c) ED length of stay, and d) CT angiography (CTA). Methods: We conducted a multicentre prospective before/after study at 6 tertiary-care EDs January 2010 to December 2016 (implementation July 2013). Consecutive alert, neurologically intact adults with a headache peaking within one hour were included. SAH was defined by subarachnoid blood on head CT (radiologists final report); xanthochromia in the cerebrospinal fluid (CSF); $>1 \times 106 / \mathrm{L}$ red blood cells in the final tube of CSF with an aneurysm on CTA. Results: We enrolled 3,669 patients, 1,743 before and 1,926 after implementation, including 185 with SAH. The investigation rate before implementation was $89.0 \%$ (range 82.9 to $95.6 \%$ ) versus $88.4 \%$ (range 85.2 to $92.3 \%$ ) after implementation. The proportion who had CT remained stable $(88.0 \%$ versus $87.4 \%$; $=0.60)$, while the proportion who had LP decreased from $38.9 \%$ to $25.9 \%(\mathrm{p}<0.001)$, and the proportion investigated with CTA increased from $18.8 \%$ to $21.6 \%(\mathrm{p}=0.036)$. The additional testing rate (i.e. LP or CTA) diminishedfrom $50.1 \%$ to $40.8 \%(\mathrm{p}<0.001)$. The proportion admitted declined from $9.8 \%$ to $7.3 \%(\mathrm{p}=0.008)$, while the mean length of ED stay was stable $(6.2+/-4.0$ to 6.4 +/- 4.1 hours; $\mathrm{p}=0.45$ ). For the 1,201 patients with CT 6 hours, there was an absolute decrease in additional testing (i.e. LP or CTA) of $15.0 \%$ (46.6\% versus $31.6 \%$; $<0.001)$. The sensitivity of the Ottawa SAH Rule was $100 \%$ (95\%CI: 98-100\%), and the 6-Hour CT Rule was 95.3\% (95\%CI: 88.998.3) for SAH. Five patients with early CT had SAH with CT reported as normal: 2 unruptured aneuryms on CTA and presumed traumatic LP (determined by treating neurosurgeon); 1 missed by the radiologist on the initial interpretation; 1 dural vein fistula (i.e. non-aneuyrsmal); and 1 profoundly anemic (Hgb 63g/L). Conclusion: The Ottawa SAH Strategy is highly sensitive and can be used routinely when SAH is being considered in alert and neurologically intact headache patients. Its implementation was associated with a decrease in LPs and admissions to hospital.

Keywords: subarachnoid hemorrhage

\section{PL02}

Probiotic regimen for outpatient gastroenteritis utility of treatment (PROGUT) study: a multicenter randomized controlled trial

S. Freedman, MD, CM, MSc, S. Williamson-Urquhart, BSc, K. Farion, MD, S. Gouin, MD, CM, A. Willan, PhD, N. Poonai, MD, MSc, K. Hurley, MD, MHI, P. Sherman, MD, Y. Finkelstein, MD, BSc,
B. Lee, MD, X. Pang, PhD, L. Chui, PhD, D. Schnadower, MD, MPH, J. Xie, MD, MPH, M. Gorelick, MD, MSCE, S. Schuh, MD, University of Calgary, Calgary, AB

Introduction: Gastroenteritis accounts for 1.7 million emergency department visits by children annually in the United States. We conducted a double-blind trial to determine whether twice daily probiotic administration for 5 days, improves outcomes. Methods: 886 children aged 348 months with gastroenteritis were enrolled in six Canadian pediatric emergency departments. Participants were randomly assigned to twice daily Lactobacillus rhamnosus R0011 and Lactobacillus helveticus R0052, $4.0 \times 109 \mathrm{CFU}$, in a 95:5 ratio or placebo. Primary outcome was development of moderate-severe disease within 14 days of randomization defined by a Modified Vesikari Scale score 9. Secondary outcomes included duration of diarrhea and vomiting, subsequent physician visits and adverse events. Results: Moderatesevere disease occurred in $108(26.1 \%)$ participants administered probiotics and $102(24.7 \%)$ participants allocated to placebo (OR 1.06; 95\% CI: 0.77, 1.46; $\mathrm{P}=0.72$ ). After adjustment for site, age, and frequency of vomiting and diarrhea, treatment assignment did not predict moderate-severe disease ( $\mathrm{OR}, 1.11,95 \% \mathrm{CI}, 0.80$ to $1.56 ; \mathrm{P}=0.53)$. In the probiotic versus placebo groups, there were no differences in the median duration of diarrhea $[52.5(18.3,95.8)$ vs. $55.5(20.2,102.3)$ hours; $\mathrm{P}=0.31$, vomiting $[17.7(0,58.6)$ vs. $18.7(0,51.6)$ hours; $\mathrm{P}=0.18$ ], physician visits $(30.2 \%$ vs. $26.6 \%$; OR $1.19 ; 95 \% \mathrm{CI} 0.87$. $1.62 ; \mathrm{P}=0.27)$, or adverse events $(32.9 \%$ vs. $36.8 \%$; OR $0.83 ; 95 \% \mathrm{CI}$ 0.62. 1.11; $\mathrm{P}=0.21$ ). Conclusion: In children presenting to an emergency department with gastroenteritis, twice daily administration of $4.0 \times 109 \mathrm{CFU}$ of a Lactobacillus rhamnosus/helveticus probiotic does not prevent development of moderate-severe disease or improvements in other outcomes measured.

Keywords: probiotic, diarrhea, pediatrics

\section{PL03}

Prophylactic administration of diphenhydramine to reduce neuroleptic side-effects in the acute care setting: a systematic review and meta-analysis

A. Mokhtari, O. Yip, J. Alain, MD, MSc, A. Turgeon, MD, MSc, S. Berthelot, MD, MSc, CHU de Québec Université Laval, Montréal, QC

Introduction: Neuroleptics are commonly used drugs to treat different conditions (e.g. psychosis, migraines) in the acute care setting and the emergency department. Their side effects can be disabling or, worse, fatal. The use of diphenhydramine to prevent those side-effects is widespread, but remains controversial. We performed a systematic review to determine if prophylactic administration of diphenhydramine (PAD) reduces the incidence of neuroleptic side-effects. Methods: Data sources: Medline, Embase, Cochrane Library, PsycInfo and Web of Science were searched. References from reviews that were identified in the search and from included studied were also reviewed for inclusion. Study selection: Randomized controlled trials evaluating any neuroleptic with PAD versus the same neuroleptic alone or with any inactive agent. Primary outcome was incidence of any extra-pyramidal sideeffect. Secondary outcomes were akathisia, usage of rescue medication, subjective restlessness, neuroleptic malignant syndrome, sedation and sedation intensity. Data extraction: Independent reviewers scanned identified citations, extracted data and assessed for risk of bias. Data analysis: Meta-analysis was performed using random effect models. Heterogeneity and quality of evidence were assessed using, respectively, I2 and the GRADE approach. Results: Results: Of 1566 identified citations, nine studies $(n=1436)$ met all eligibility criteria. Four studies 
were specifically designed to assess for neuroleptic side-effects. Four studies were at high risk of bias. In primary analysis, PAD had no effect on the incidence of extra-pyramidal symptoms ( 7 studies, $n=1393$ patients, RR 0.70 [0.40-1.22]), akathisia (5 studies, $\mathrm{n}=1094$ patients, RR 0.81 [0.36-1.82]) and sedation (5 studies; $\mathrm{n}=1079$, RR 1,48 [0.902.42]). Higher dosage of diphenhydramine was not associated with a greater reduction of extra-pyramidal side-effects. In a sensitivity analysis excluding an outlier study $(\mathrm{n}=120$, RR 6.63 [1.55-28,35]), PAD was associated with a significant decrease in extra-pyramidal side-effects (6 studies, $\mathrm{n}=1273$, RR 0.56 [0,38-0.82]), but not with any of the secondary outcome measures. Conclusion: Conclusion: When excluding an outlier study, PAD was associated with a significant reduction of extra-pyramidal side-effects. However, PAD did not significantly influence the incidence of akathisia. Overall quality of evidence is low. Further studies are warranted. PAD represents an interesting treatment option against neuroleptic side-effects, but its widespread usage whitout strong evidence to support it raises concerns. Keywords: neuroleptic side-effects, diphenhydramine, systematic review

\section{PL04}

Effectiveness of hospital avoidance interventions among elderly patients: a systematic review

A. Ness, MD, N. Symonds, M. Siarkowski, BSc, MBT, M. Broadfoot, K. McBrien, MD, MPH, E. S. Lang, MD, CM, J. Holroyd-Leduc, MD, P. Ronksley, PhD, University of Calgary, Calgary, $\mathrm{AB}$

Introduction: Overuse of acute care services, particularly emergency department (ED) use, is an important topic for healthcare providers and policy makers within Canada and abroad. Prior work has shown that frail elderly patients with complex medical needs and limited personal and social resources are heavy users of ED services and are often admitted when they present to the ED. Updated information on the most effective strategies to avert ED presentation and hospital admission focused specifically on elderly patients is needed. Methods: This systematic review addressed the question: what interventions have demonstrated effectiveness in decreasing ED use and hospital admissions in elderly patients? Comprehensive literature searches were conducted in databases including Ovid Medline, EMBASE, CINAHL, and the Cochrane Central Register of Controlled Trials with no language or date restrictions. Citations were limited to interventional studies. Grey literature and reference list searches, as well as communication with experts in the field were performed. Consensus or a third reviewer resolved any disagreements. Original research regarding interventions conducted in populations 65 years or older with acute illness, either living in community or facility-living were included. Primary outcomes were ED visits and hospital admissions. Secondary outcomes included: mortality, cost, and patient-reported outcomes such as health-related quality of life and functional status. Results: Forty-three relevant studies were identified including 22 randomized controlled trials (RCT), 2 cluster-RCT, 2 trials with non-random allocation, 4 before-after studies, 6 quasi-experimental studies, and 7 cohort studies. Intervention settings included: home visits (22), long-term care (7), outpatient or primary care clinics (8), and ED (3) or inpatient (3). Data characterization revealed that home-based, outpatient and/or primary carebased strategies reduced ED visits and hospitalizations, particularly those which included comprehensive geriatric assessments, home visits or regular face-to-face contact and interdisciplinary teams. Hospital-based models generally showed no difference in ED or inpatient service utilization. There was, however, considerable variability across individual studies with respect to reporting of outcomes, statistical analyses performed, and overall risk of bias. Conclusion: Various interventional strategies have been studied to avert ED presentation and hospital admission for frail elderly patients. More rigorous methodology and standardization of outcome measures is needed to quantitatively assess the effects of these programs.

Keywords: elderly, emergency department avoidance, systematic review

\section{Oral Presentations}

\section{LO01}

Analysis of bystander CPR quality during out-of-hospital cardiac arrest using data derived from automated external defibrillators

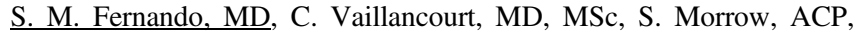
I. G. Stiell, MD, MSc, University of Ottawa, Department of Emergency Medicine, Ottawa, ON

Introduction: Out-of-hospital cardiac arrest (OHCA) is associated with high mortality, and CPR quality is one of the few modifiable factors associated with improved outcomes. Particularly, bystander CPR has been shown to improve survival and neurological outcomes in OHCA. However, the quality of CPR performed by bystanders in OHCA is unknown. We evaluated bystander CPR quality during OHCA, utilizing data stored within Automated External Defibrillators (AEDs), and matched with cases enrolled in the Resuscitation Outcomes Consortium (ROC) database. Methods: This cohort study included adult OHCA cases from the Ottawa ROC site between 2011-2016, which were of presumed cardiac etiology, not witnessed by EMS, and where an AED was utilized by a bystander with $>1$ minute of CPR process data available. AED data from Ottawa Paramedic Services was matched to each case identified by the ROC database. AED data was analyzed using manufacturer software to determine overall measures of bystander CPR quality, changes in bystander CPR quality over time, and bystander adherence to existing 2010 Resuscitation Guidelines. Results: 100 cases met all inclusion criteria. $75.0 \%$ of patients were male, with a mean age of 62.3 years. $58.0 \%$ of arrests occurred in the home setting, and $24.0 \%$ were witnessed arrests. Initial rhythm was ventricular fibrillation/ventricular tachycardia in $36.0 \%$ of cases. Overall survival rate was $42.0 \%$, with a modified Rankin Score of 3.7 (95\% CI: 2.9-4.5). Bystanders demonstrated high-quality CPR over the course of resuscitation, with a chest compression fraction (CCF) of $75.9 \%$ (73.6-78.1), a compression depth of $5.26 \mathrm{~cm}$ (5.03-5.49), and a compression rate of 111.2/min (107.7-114.7). Mean peri-shock pause was 26.8 seconds (24.6-29.1). Adherence rates to 2010 Resuscitation Guidelines for compression rate and depth were $66.0 \%$ (60.9-71.1) and 54.9\% (48.6-61.3), respectively. CPR quality was lowest in the first minute of resuscitation, during which rhythm analysis took place (mean $40.5 \mathrm{sec}$ ). In cases involving a shockable rhythm, overall latency from initiation of AED to shock delivery was $59.2 \mathrm{sec}$ (45.5-72.8). Conclusion: We found that bystanders perform high-quality CPR, with strong adherence rates to existing Resuscitation Guidelines. Our findings provide evidence of the quality of bystander CPR performed during OHCA.

Keywords: cardiac arrest, cardiopulmonary resuscitation, bystander cardiopulmonary resuscitation

\section{LO02}

Characteristics and predictors of pediatric emergency department use in Manitoba: a population based study

L. K. Crockett, MSc, E. Wall-Wieler, MSc, T. Klassen, MD, MSc, George and Fay Yee Centre for Health Care Innovation, Winnipeg, MB

Introduction: Within Manitoba, little is known about the current state of pediatric emergency department (ED) use or the state of provincial 